\title{
Correction to: Curcumin: a modulator of inflammatory signaling pathways in the immune system
}

\author{
Kolsoum Rezaie Kahkhaie ${ }^{1,2}$. Ali Mirhosseini ${ }^{3}$. Ali Aliabadi ${ }^{4}$. Asadollah Mohammadi ${ }^{5}$. \\ Mohammad Javad Mousavi ${ }^{6,7}$. Saeed Mohammadian Haftcheshmeh ${ }^{8} \cdot$ Thozhukat Sathyapalan $^{9}$. \\ Amirhossein Sahebkar ${ }^{10,11,12}$ (1)
}

Published online: 19 August 2019

๑) Springer Nature Switzerland AG 2019

\section{Correction to: Infammopharmacology https://doi.org/10.1007/s10787-019-00607-3}

Unfortunately, the 4th author name was incorrectly published in the original article. The complete correct name is given below.

Mohammad Javad Mousavi.

The original article has been updated.

The original article can be found online at https://doi.org/10.1007/ s10787-019-00607-3.

Saeed Mohammadian Haftcheshmeh

Mohammadianhs941@mums.ac.ir; saeedmoh69@gmail.com

$\triangle$ Amirhossein Sahebkar

sahebkara@mums.ac.ir; amir_saheb2000@yahoo.com

1 Department of Medical Biochemistry, Faculty of Medicine, Zabol University of Medical Sciences, Zabol, Iran

2 Medicinal Plants Research Center, Faculty of Medicine, Zabol University of Medical Sciences, Zabol, Iran

3 Immunology Research Center, Inflammation and Inflammatory Diseases Division, School of Medicine, Mashhad University of Medical Sciences, Mashhad, Iran

4 Department of Medicinal Chemistry, School of Pharmacy, Mashhad University of Medical Sciences, Mashhad, Iran

5 Cellular and Molecular Research Center, Research Institute for Health Development, Kurdistan University of Medical Sciences, Sanandaj, Iran

6 Department of Immunology and Allergy, Faculty of Medicine, Bushehr University of Medical Sciences, Bushehr, Iran
Publisher's Note Springer Nature remains neutral with regard to jurisdictional claims in published maps and institutional affiliations.
7 Immunology Department, School of Medicine, Tehran University of Medical Sciences, Tehran, Iran

8 Department of Medical Immunology, Faculty of Medicine, Mashhad University of Medical Sciences, Mashhad 91766-99199, Iran

9 Department of Academic Diabetes, Endocrinology and Metabolism, Hull York Medical School, University of Hull, Hull HU3 2JZ, UK

10 Biotechnology Research Center, Pharmaceutical Technology Institute, Mashhad University of Medical Sciences, Mashhad, Iran

11 Neurogenic Inflammation Research Center, Mashhad University of Medical Sciences, Mashhad, Iran

12 Department of Medical Biotechnology, School of Medicine, Mashhad University of Medical Sciences, Mashhad 91779-48564, Iran 